NASA Technical Memorandum 87154

\title{
A Unique Set of Micromechanics Equations for High Temperature Metal Matrix Composites
}

Dale A. Hopkins and Christos C. Chamis Lewis Research Center

Cleveland, Ohio

Prepared for the

First Symposium on Testing Technology of Metal Matrix Composites sponsored by the American Society for Testing and Materials Nashville, Tennessee, November 18-20, 1985 


\title{
A UNIQUE SET OF MICROMECHANICS EQUATIONS FOR HIGH TEMPERATURE
}

\section{METAL MATRIX COMPOSITES}

Dale A. Hopkins and Christos C. Chamis

\section{National Aeronautics and Space Administration}

Lewis Research Center

Cleveland, Ohio 44735

\begin{abstract}
A unique set of micromechanics equations is presented for high temperature metal matrix composites. The set includes expressions to predict mechanical properties, thermal properties, and constituent microstresses for the unidirectional fiber reinforced ply. The equations are derived based on a mechanics of materials formulation assuming a square array unit cell model of a single fiber, surrounding matrix and an interphase to account for the chemical reaction which commonly occurs between fiber and matrix. A preliminary validation of the equations was performed using three-dimensional finite element analysis. The results demonstrate excellent agreement between properties predicted using the micromechanics equations and properties simulated by the finite element analyses. Implementation of the micromechanics equations as part of an integrated computational capability for nonlinear structural analysis of high temperature multilayered fiber composites is 11lustrated.
\end{abstract}

Key Words: Metal matrix composites; Composite micromechanics; Mechanical properties; Thermal properties; Uniaxial strengths; Microstresses 


\section{Introduction}

The mechanical performance and structural integrity of fiber reinforced metal matrix composites are ultimately governed by the behavior of the constituent materials at a micromechanistic level. In general, the individual constituents behave quite differently relative to one another. Moreover, behavior of the constituents is dynamic, particularly in high temperature applications, due to the various nonlinearities assoclated with, for example:

(1) large local stress excursions, (2) temperature-dependent material properties, (3) time-dependent effects, and (4) constituent chemical reaction.

In the structural analys is of metal matrix composites, then, it is important to be able to describe and track this micromechanistic constituent behavior. Avallable methods for this purpose are limited. For example, techniques such as finite element analysis can, in principle, be applied directly with the constituents modeled discretely. It becomes obvious, however, that for complex structures the resources (manpower and computer) necessary to define, conduct and interpret such an analysis are prohibitive. Another approach is to employ composite micromechanics theory and derive simplified relationships which describe the three-dimensional anisotropic behavior of the simple composite (e.g., unidirectional ply). The latter approach has been taken as part of a comprehensive research program to develop effective computational mechanics methodologies for high temperature multilayered fiber composite structures.

As an essential part of the above-mentioned program, a unique set of micromechanics equations has been derived for high temperature metal matrix composites. The set comprises closed-form expressions to predict equivalent "pseudo homogeneous" properties for the unidirectional fiber reinforced ply, including: (1) mechanical properties - moduli, Poisson's ratios, and uniaxial 
strengths; (2) thermal properties - conductivities, coefficients of expansion, and heat capacity; and (3) constituent microstresses.

The micromechanics equations presented here are derived based on a mechanics of materials formulation assuming a square array unit cell model of a single fiber, surrounding matrix and an interphase to account for the chemical reaction which commoniy occurs between fiber and matrix. The basis of the formulation is summarized as part of the discussion below.

Concurrent with the derivation of equations, a study was conducted using three-dimensional finite element anaiysis. The purpose of the study was to assess the validity of the mechanics of materials formulation, in general, and to investigate the accuracy of the micromechanics equations for a specific composite material system. Results from this study are presented also as part of the discussion below.

Finally, a demonstration of the utility of this unique set of micromechanics equations is provided by illustrating their use as part of an integrated computational capability for the nonlinear structural analysis of high temperature multilayered fiber composites. A few typical results are presented from the stress analysis of a hypothetical tungsten fiber reinforced superalloy turbine airfoil.

Composite Micromechanics Theory

Composite micromechanics theory refers to the collection of physical principles, mathematical models, assumptions and approximations employed to relate the behavior of a simple composite unit (e.g., lamina or ply) to the behavior of its individual constituents. For example, a variety of approaches have been used in the past to predict equivalent thermoelastic material properties of unidirectional fiber composites [1-6]. More recently, simple equations have been derived $[7,8]$ to predict mechanical, thermal, and strength properties for resin matrix composites using a mechanics of materials 
formulation. A similar approach was taken to derive the set of micromechanics equations presented here for high temperature metal matrix composites.

The formal procedure of composite micromechanics theory relies on the principles of solid mechanics, thermodynamics, etc., at different levels of mathematical sophistication, together with certain assumptions (consistent with the physical situation) and approximations. In the approach taken here, application is made of the principles of displacement compatibility and force equilibrium as defined in elementary mechanics-of-materials theory and Fourier's law for heat conduction from thermodynamics. In addition, the assumptions are made that: (1) fibers are continuous and parallel; (2) properties of all fibers are identical; and (3) complete bonding exists between constituents. No restrictions need be placed on the constitutive behavior or isotropy of the individual constituent materials. For generality, constituent material behavior can be taken as thermoviscoplastic, anisotropic, and three-dimensional. It is implied by this that the individual constituent material histories can be tracked independentiy as a function of time and represented as an instantaneous stress/strain state.

The periodic structure of a unidirectional metal matrix composite (ply) is approximated here by a square array unit cell model. The geometry of the model is illustrated in Fig. 1. It should be noted that the interphase growth is assumed to result from the degradation of fiber material and thus propagates inward causing a continuous decrease of the current (intact) fiber diameter (D) from the original (virgin) fiber diameter $\left(D_{0}\right)$. With the existence of the interphase, three subregions $(A, B, C)$ are distinguished to characterize the intralaminar (through-the-thickness) nonuniformity of the constituent (matrix and interphase) microstresses and material properties.

The definition of ply properties is with respect to the ply material coordinate system which is depicted in Fig. 2. The common terminology 
associated with each of the coordinate axis directions is also illustrated on the ply schematic. The micromechanics equations presented here are derived for the special case of a transversely isotropic (isotropic in the 2-3 plane) ply allowing for transversely isotropic constituents.

\section{Composite Micromechanics Equations}

The micromechanics equations to predict ply equivalent mechanical properties are summarized in Fig. 3. Included are expressions for normal (extensional) moduli ( $\left.E_{\ell 11}, E_{\ell 22}\right)$, shear moduli $\left(G_{\ell 12}, G_{\ell 23}\right)$, and Poisson's ratios $\left(v_{\ell 12}, v_{223}\right)$. In the expressions $k$ represents constituent original volume fraction (values prior to any interphase growth) and the subscripts $f$, $m, d$, and $\ell$ denote fiber, matrix, interphase, and ply quantity, respectively. The volume fraction of interphase is expressed in terms of the fiber original volume fraction and the virgin and intact (in situ) fiber diameters.

The equations for moduli are derived with modulus taken in the general context as simply the derivative of stress with respect to strain. As such, the expressions are applicable to the prediction of instantaneous or tangent moduli as well as elastic moduli. It should be noted that the expressions for transverse moduli do not account for the longitudinal Poisson restraining effect that the fiber imparts on the matrix. The restrained matrix effect is considered here to be negligible for metal matrix composites.

The effect is generally more significant in resin matrix composites, for example, where the fiber/matrix relative stiffness ratio is much greater.

The ply equivalent thermal properties are predicted by the micromechanics equations summarized in Fig. 4. Included are expressions for heat capacity $\left(C_{\ell}\right)$, thermal conductivities $\left(K_{\ell 11}, K_{\ell 22}\right)$, and thermal expansion coefficients $\left(\alpha_{\ell 11}, \alpha_{\ell 22}\right)$. In the expression for heat capacity the symbol. $\rho$ represents density. 
The ply in-plane unfaxial strengths are predicted by the micromechanics equations summarized in Figs. 5 and 6 . Included are expressions for tensile strength $\left(S_{\ell 11 T}, S_{\ell 22 T}\right)$, compressive strength $\left(S_{\ell 11 C}, S_{\ell 22 C}\right)$, and intralaminar shear strength $\left(S_{\ell 12 S}\right)$. Each of the ply strengths is associated with a specific fallure mode, as illustrated by the schematics in Fig. 7. In the case of longitudinal compressive strength, four different fallure modes are considered. The four expressions in Fig. 5 for $S_{\ell l l C}$ correspond, respectively, to the four fallure modes as follows; fiber compression mode, matrix compression mode, delamination/splitting mode, and fiber microbuckling mode. A more comprehensive treatment of micromechanics strength theories is given by Chamis [9].

The expressions to predict the thermomechanical microstress distribution in the ply constituents are summarized in Figs. 8 to 10 . Included are expressions for fiber microstresses $\left(\sigma_{f 11}, \sigma_{f 22}, \sigma_{f 12}, \sigma_{f 23}\right)$ interphase microstresses $\left(\sigma_{\mathrm{d} 11}, \sigma_{\mathrm{d} 22}^{B, C}, \sigma_{\mathrm{d} 12}^{B, C}, \sigma_{\mathrm{d} 23}^{B, C}\right)$ and matrix microstresses $\left(\sigma_{\mathrm{m} 11}\right.$, $\left.\sigma_{m 22}^{A, B, C}, \sigma_{m i 2}^{A, B, C}, \sigma_{m 23}^{A, B, C}\right)$. In the expressions $\Delta T$ represents an incremental change in temperature and the superscripts A, B, and C denote the intralaminar subregions 11 lustrated in the accompanying schematics. It should be noted that these expressions for constituent microstresses are based on uniaxial behavior, i.e., they do not incorporate any Poisson contributions.

The systematic procedure for deriving the micromechanics equations summarized above is explicitiy demonstrated in the Appendix with the derivations for normal modult $\left(E_{\ell 11}\right.$ and $\left.E_{\ell 22}\right)$. Derivations of the other equations are omitted here solely for the sake of brevity. The selection of $E_{\ell 11}$ and $E_{\ell 22}$ for demonstration purposes was based on the authors' judgment that their derivations are sufficiently representative to adequately demonstrate the formal procedure. 
Micromechanics/Finite Element Validation

In order to investigate the validity of the mechanics of materials formulation and assess the accuracy of the equations derived therefrom, a preliminary study was conducted using three-dimensional finite element analysis. The objective of the study was to compare the equivalent ply properties ( $E_{\ell 71}, E_{\ell 22}, G_{\ell 12}, G_{\ell 23}, v_{\ell 12}, v_{\ell 23}, \alpha_{\ell 17}, \alpha_{\ell 22}$ ) predicted by the micromechanics equations with the average "pseudo homogeneous" ply properties simulated in the finite element analyses.

To conduct the analyses, a discrete model of the square array unit cell was constructed, as shown in Fig. 11, from isoparametric solid finite elements. The composite material system assumed for this study involved a thoriated tungsten $\left(\mathrm{W}-1.5 \mathrm{ThO}_{2}\right)$ fiber embedded in an iron-base superalloy (Fe-25Cr-4Al-1Y) matrix. Properties for the interphase were taken to be a simple average of the flber and matrix properties.

The analyses entailed simulations of idealized modes of deformation such as simple elongation, pure shear, and unconstrained thermal expansion. These were achieved through the judicious application of the loading/boundary conditions on the model. The appropriate simple expressions from elementary mechanics of materials theory (see Fig. 11) were then applied in conjunction with the nodal displacement/force results of the finite element analyses to compute the simulated average properties of the discrete model as a "pseudo homogeneous" unit.

Results of the study are summarized in Table 1 which gives the ratios of property values determined from the micromechanics equations ( $P_{M E Q}$ ) and by finite element simulation $\left(P_{F E M}\right)$. As can be seen, excellent agreement was achieved overall. These results indicate that the mechanics of materials formulation is an effective approach to the micromechanical modeling of metal 
matrix composites. It is recognized, however, that additional investigation, both analytical and experimental, would be prudent before any final conclusions are made regarding the specific accuracy of these micromechanics equations.

Application of Micromechanics Equations

The primary impetus in deriving the set of micromechanics equations presented here was for implementation as part of an integrated computational capability for the nonlinear analysis of high temperature multilayered fiber composites [10]. This particular utilization of the equations is demonstrated here with a few typical results taken from the nonlinear (quasi-static) stress analysis of a hypothetical turbine blade (airfoil only) model. The incremental/iterative analysis was conducted to investigate the thermally induced residual stresses developed during the cool-down transient of a typical fabrication process.

The airfoil is a hollow thin shell structure of constant thickness with walls comprising a four-ply $[ \pm 45]_{S}$ laminate based on $\mathrm{W}-1.5 \mathrm{ThO}_{2}$ fiber reinforced $\mathrm{Fe}-25 \mathrm{Cr}-4 \mathrm{Al}-\mathrm{TY}$ at a fiber volume fraction of 0.50 . Since the purpose here is merely to illustrate the types of information provided by the micromechanics equations in this particular implementation, further details of the airfoil model and analysis are omitted.

Two examples of ply mechanical property predictions are given in figs. 12 and 13 which show the variation during the cool-down transient of constituent and ply longitudinal and transverse moduli, respectively. The ply moduli are computed from the corresponding micromechanics equations. The results in Fig. 12 reflect the rule-of-mixtures relationship expressed by the equation for $E_{\text {Q11 }}$ while the results in Fig. 13 11lustrate the dominance of the matrix modulus on the value for $E_{\ell 22}$. 
The development of residual stresses during the cool-down transient is illustrated in Figs. 14 and 15. The results are for the longitudinal and transverse normal components, respectively, of ply stress and constituent microstresses. The microstresses are computed from the corresponding micromechanics equations. The points to be noted from these results are the relative magnitudes and sense (tensile or compressive) of the constituent microstresses. In Fig. 14, for example, the opposite sense of the fiber and matrix microstresses results from the difference in thermal expansion coefficients between the two materials. The results in Fig. 15 illustrate the significant through-the-thickness nonuniformity of the matrix and interphase microstresses, as characterized in the different intralaminar subregions $(A, B, C)$.

From just the few examples given, the utility of the micromechanics equations becomes more apparent. Considering the results of microstress distribution, for example, it becomes intuitively more clear how material fallures might occur at a local level and prompt the initiation of a flaw. This type of information provides an insight into the behavior of composites at a micromechanistic level which undoubtedly influences their performance and integrity in a structural application.

Summary

The set of micromechanics equations presented here for high temperature metal matrix composites includes expressions to predict the mechanical properties, thermal properties, and constituent microstress distribution for a unidirectional fiber reinforced ply. The equations incorporate an interphase region at the fiber/matrix boundary in order to account for the chemical reaction which commonly occurs in high temperature applications of these composites. The basis of the mechanics of materials formulation from which the equations are derived is described. The formulation is shown to be a valid 
and effective approach to micromechanical modeling of metal matrix composites, supported by the favorable results achieved in a comparison with three-dimensional finite element analysis. The utility of the micromechanics equations as part of an integrated composite structural analysis capability is illustrated with examples taken from the nonlinear stress analysis of a turbine airfotl. The results demonstrate the ability to describe and track behavior at a micromechanistic level which impacts the performance and integrity of these composites in structural applications. 


\section{Appendix}

In order to demonstrate the formal procedure involved in the application of composite micromechanics theory, derivations of the equations for ply normal moduli ( $E_{\ell 11}$ and $E_{\ell 22}$ ) are explicitly developed below. The particular approach taken here relies on the principles of force equilibrium and displacement compatibility as defined from elementary mechanics-of-materials theory.

Longitudinal Normal Modulus

Consider the square array unit cell model (see Fig. 1) subjected to a uniaxial load in the longitudinal direction (see Fig. 2). The equivalent composite ( $p l y$ ) load is defined from force equilibrium to be the sum of the constituent loads as follows:

$$
P_{\ell}=P_{f}+P_{d}+P_{m}
$$

In the integrated average sense, Eq. (1) is rewritten as

$$
\sigma_{\ell} A_{\ell}=\sigma_{f} A_{f}+\sigma_{d} A_{d}+\sigma_{m} A_{m}
$$

where $A$ represents cross-sectional area. Dividing through by $A_{\ell}$ and noting that because of a common longitudinal dimension the resulting area ratios are equivalent to actual volume fractions. Eq. (2) reduces to

$$
\sigma_{\ell}=\sigma_{f} k_{f}^{\prime}+\sigma_{d} k_{d}^{\prime}+\sigma_{m} k_{m}^{\prime}
$$

Because compatibility of longitudinal displacement requires equal strains for the composite and constituents $\left(\varepsilon_{l}=\varepsilon_{f}=\varepsilon_{d}=\varepsilon_{m}\right)$, Eq. (3) can be differentiated with respect to strain to give

$$
\left(\frac{d \sigma_{\ell}}{d \varepsilon}\right)=\left(\frac{d \sigma_{f}}{d \varepsilon}\right) k_{f}^{\prime}+\left(\frac{d \sigma_{d}}{d \varepsilon}\right) k_{d}^{\prime}+\left(\frac{d \sigma_{m}}{d \varepsilon}\right) k_{m}^{\prime}
$$

The quantities $(d \sigma / d \varepsilon)$ represent the slopes of the corresponding stressstrain curves for the composite and constituents and in this context define instantaneous or "tangent" modul1. Hence, Eq. (4) becomes

$$
E_{\ell}=E_{f} k_{f}^{\prime}+E_{d} k_{d}^{\prime}+E_{m} k_{m}^{\prime}
$$


Expressing actual volume fractions in terms of original fiber and matrix volume fractions (before interphase growth) and original and intact fiber diameters, Eq. (5) is rewritten as

$$
E_{\ell}=k_{f}\left\{\left(\frac{D}{D_{0}}\right)^{2} E_{f}+\left[1-\left(\frac{D}{D_{0}}\right)^{2}\right] E_{d}\right\}+k_{m} E_{m}
$$

Equation (6) is the desired form and is the same as that given in Fig. 3 .

\section{Transverse Normal Modulus}

Consider the square array unit cell model again except that the fiber and interphase are of equivalent square cross-section such that linear dimensions (in the plane of cross-section) can be defined as follows:

$$
a_{f}=\left(\frac{\pi}{4}\right)^{1 / 2} D_{,} \quad a_{d}=\left(\frac{\pi}{4}\right)^{1 / 2} D_{0}, \quad a_{\ell}=\left(\frac{\pi}{4 k_{f}}\right)^{1 / 2} D_{0}
$$

and

$$
s_{f}=a_{f}, \quad s_{d}=a_{d}-a_{f}, \quad s_{m}=a_{\ell}-a_{d}, \quad s_{\ell}=a_{\ell}
$$

Assume a uniaxial load in the transverse direction and neglect Poisson effects. For subregion C displacement compatibility yields

$$
s_{\ell} \varepsilon_{\ell}=s_{f} \varepsilon_{f}+s_{d} \varepsilon_{d}+s_{m} \varepsilon_{m}
$$

and force equilibrium results in equal stresses for the composite and constituents $\left(\sigma_{\ell}=\sigma_{f}=\sigma_{d}=\sigma_{m}\right)$. Hence, eq. (9) can be differentiated with respect to stress to give

$$
\left(\frac{d \varepsilon_{\ell}}{d \sigma}\right) s_{\ell}=\left(\frac{d \varepsilon_{f}}{d \sigma}\right) s_{f}+\left(\frac{d \varepsilon_{d}}{d \sigma}\right) s_{d}+\left(\frac{d \varepsilon_{m}}{d \sigma}\right) s_{m}
$$

The quantities $(d \varepsilon / d \sigma)$ represent reciprocals of the slopes of the corresponding stress-strain curves for the composite and constituents and in the same context as before define reciprocals of instantaneous or "tangent" modulf. Hence, with some rearranging Eq. (10) becomes 


$$
E_{\ell}^{C}=\frac{E_{m}}{\left[\left(\frac{s_{m}}{s_{\ell}}\right)+\left(\frac{s_{d}}{s_{\ell}}\right)\left(\frac{E_{m}}{E_{d}}\right)+\left(\frac{s_{f}}{s_{\ell}}\right)\left(\frac{E_{m}}{E_{f}}\right)\right]}
$$

Substituting the definitions in Eqs. (7) and (8) into Eq. (11) and rearranging gives

$$
E_{l}^{C}=\frac{E_{m}}{\left\{1-\sqrt{k_{f}}\left[\left(1-\frac{D}{D_{0}}\right)\left(\frac{E_{m}}{E_{d}}\right)-\left(\frac{D}{D_{0}}\right)\left(\frac{E_{m}}{E_{f}}\right)\right]\right\}}
$$

which defines an equivalent modulus for subregion $C$. The equivalent modulus for subregion $B$ is deduced from Eq. (12) by letting $D / D_{0}$ equal unity. The result is

$$
E_{\ell}^{B}=\frac{E_{m}}{\left\{1-\sqrt{k_{f}}\left[1-\left(\frac{E_{m}}{E_{f}}\right)\right]\right\}}
$$

The equivalent modulus for subregion $A$ is simply the matrix modulus or

$$
E_{\ell}^{A}=E_{m}
$$

The ply transverse modulus ( $E_{\ell 22}$ ), then, is defined by assuming that subregions $A, B$, and $C$ act as parallel elements when subjected to a transverse load. This is analogous to the case for $E_{\ell 11}$ where the constituents are assumed to act in parallel. Hence, from Eq. (5)' it is deduced that

$$
E_{\ell} s_{\ell}=E_{\ell}^{C_{f}}+E_{\ell}^{B} s_{d}+E_{\ell}^{A} s_{m}
$$

Dividing through by $s_{\ell}$, substituting the definitions from Eqs. (7) and (8) and the results from Eqs. (13) through (15), and rearranging gives

$$
E_{\ell}=E_{m}\left\{\left(1-k_{f}\right)+\frac{\sqrt{k_{f}}\left[1-\left(\frac{D}{D_{0}}\right)\right]}{1-\sqrt{k_{f}}\left[1-\left(\frac{E^{m}}{E_{f}}\right)\right]}+\frac{\sqrt{k_{f}}\left(\frac{D}{D_{0}}\right)}{1-\sqrt{k_{f}}\left[1-\left(1-\frac{D}{D_{0}}\right)\left(\frac{E_{m}}{E_{d}}\right)-\left(\frac{D}{D_{0}}\right)\left(\frac{E_{m}}{E_{f}}\right)\right]}\right\}
$$

Equation (16) is the desired form and is the same as that given in Fig. 3. 
1. Chamis, C.C., and Sendeckyj, G.P., Journal of Composite Materials, Vo1. 2, No. 3, Ju1y 1968, pp. 332-358.

2. Ekva11, J.C., "Elastic Properties of Orthotropic Monofilament Laminates," ASME Paper 61-AV-56, ASME Aviation Conference, Los Angeles, CA, 1961.

3. Abolin'sh, D.S., Polymer Mechanics, Vol. 1, No. 4, July-Aug. 1965, pp. 28-32.

4. Springer, G.S. and Tsai, S.W., Journal of Composite Materials, Vol. 1, No. 2, Apr. 1967, pp. 166-173.

5. Agarwa 1, B.D. and Broutman, L.J., Analysis and Performance of Fiber Composites, Wiley, New York, 1980.

6. Halpin, J.C., Primer on Composite Materials: Analysis, 1st ed. revised, Technomic, Lancaster, PA, 1984.

7. Chamis, C.C., SAMPE QUARTERLY, Vo1. 15, No. 3, Apr. 1984, pp. 14-23.

8. Chamis, C.C., SAMPE QUARTERLY, Vo1. 15, No. 4, July 1984, pp. 41-55.

9. Chamis, C.C., in Fracture and Fatigue, L.J. Broutman, Ed., Academic Press, New York, 1974, pp. 94-148.

10. Hopkins, D.A., "Nonlinear Analysis for High Temperature Multilayered Fiber Composite Structures," NASA TM-83754, National Aeronautics and Space Administration, Washington, DC, 1984. 
TABLE 1. - MICROMECHANICS/

FINITE ELEMENT VALIDATION;

COMPARISON OF PROPERTY

PREDICTIONS/

SIMULATIONS

\begin{tabular}{|c|c|}
\hline Property & $P_{M E Q} / P_{F E M}$ \\
\hline$E_{\ell 11}$ & 1.00 \\
$E_{\rho 22}$ & 1.01 \\
$G_{\ell 12}$ & .96 \\
$G_{\ell 23}$ & .98 \\
$v_{\ell 12}$ & 1.00 \\
$v_{\ell 23}$ & 1.08 \\
$\alpha_{\ell 11}$ & .99 \\
$\alpha_{\ell 22}$ & 1.15 \\
\hline
\end{tabular}

PMEO - Property

predicted by

micromechanics

equation.

PFEM - Property

simulated by finite

element analysis. 


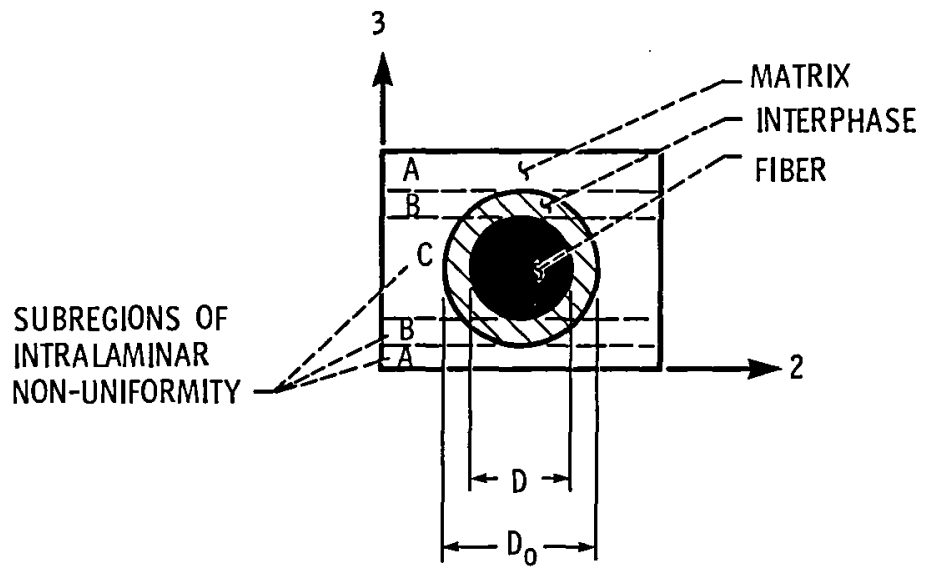

Figure 1. - Micromechanics model; square array unit cell.

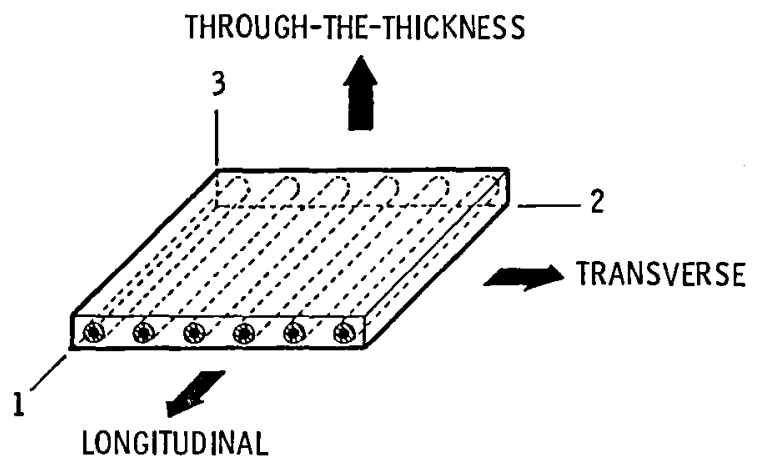

Figure 2 - Unidirectional composite (ply) material coordinate system. 


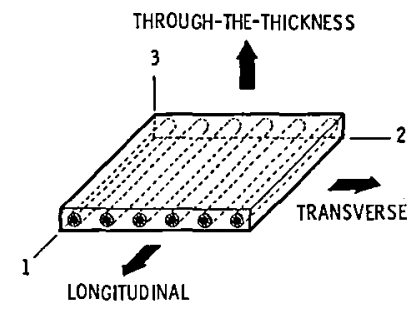

$$
\begin{aligned}
& E_{l 1 l}=k_{m} E_{m l l}+k_{f}\left\{\left[1-\left(\frac{D}{D_{0}}\right)^{2}\right] E_{d l l}+\left(\frac{D}{D_{0}}\right)^{2} E_{f l l}\right\} \\
& E_{\ell 22}=E_{m 22}\left\{\left(1-\sqrt{k_{f}}\right)+\frac{\sqrt{k_{f}}\left(1-\frac{D}{D_{0}}\right)}{1-\sqrt{k_{f}}\left(1-\frac{E_{m 22}}{E_{d 22}}\right)}+\frac{\sqrt{k_{f}}\left(\frac{D}{D_{0}}\right)}{1-\sqrt{k_{f}}\left[1-\left(1-\frac{D}{D_{0}}\right) \frac{E_{m 22}}{E_{d 22}}-\left(\frac{D}{D_{0}}\right) \frac{E_{m 22}}{E_{f 22}}\right]}\right\} E_{\ell 33} \\
& G_{\ell 12}=G_{m 12}\left\{\left(1-\sqrt{k_{f}}\right)+\frac{\sqrt{k_{f}}\left(1-\frac{D}{D_{0}}\right)}{1-\sqrt{k_{f}}\left(1-\frac{G_{m 12}}{G_{d 12}}\right)}+\frac{\sqrt{k_{f}}\left(\frac{D}{D_{0}}\right)}{1-\sqrt{k_{f}}\left[1-\left(1-\frac{D}{D_{0}}\right) \frac{G_{m 12}}{G_{d 12}}-\left(\frac{D}{D_{0}}\right) \frac{G_{m 12}}{G_{f 12}}\right]}\right\} G_{\ell 13} . \\
& G_{\ell 23}=G_{m 23}\left\{\left(1-\sqrt{k_{f}}\right)+\frac{\sqrt{k_{f}}\left(1-\frac{D}{D_{0}}\right)}{1-\sqrt{k_{f}}\left(1-\frac{G_{m 23}}{G_{d 23}}\right)}+\frac{\sqrt{k_{f}}\left(\frac{D}{D_{0}}\right)}{1-\sqrt{k_{f}}\left[1-\left(1-\frac{D}{D_{0}}\right) \frac{G_{m 23}}{G_{d 23}}-\left(\frac{D}{D_{0}}\right) \frac{G_{m 23}}{G_{f 23}}\right]}\right\} \\
& u_{\ell 12}=k_{m} u_{m 12}+k_{f}\left\{\left[l-\left(\frac{D}{D_{0}}\right)^{2}\right] u_{d 12}+\left(\frac{D}{D_{0}}\right)^{2} u_{f 12}\right\}=v_{\ell 13} \\
& U_{\ell 23}=\frac{E_{\ell 22}}{2 G_{\ell 23}}-1
\end{aligned}
$$

Figure 3. - Micromechanics equations; ply mechanical properties. 


$$
\begin{aligned}
& \text { THROUGH-THE-THICKNESS } \\
& c_{\ell}=k_{m}\left(\frac{\rho_{m}}{P_{l}}\right) c_{m}+k_{f}\left\{\left[1-\left(\frac{D}{D_{0}}\right)^{2}\right]\left(\frac{\rho_{d}}{P_{l}}\right) c_{d}+\left(\frac{D}{D_{0}}\right)^{2}\left(\frac{\rho_{f}}{P_{l}}\right) c_{f}\right\} \\
& K_{\ell 11}-k_{m} K_{m 11}+k_{f}\left\{\left[1-\left(\frac{D}{D_{0}}\right)^{2}\right] K_{d 11}+\left(\frac{D}{D_{0}}\right)^{2} K_{f 11}\right\} \\
& K_{\ell 22}=K_{m 22}\left\{\left(1-\sqrt{k_{f}}\right)+\frac{\sqrt{k_{f}}\left(1-\frac{D}{D_{0}}\right)}{1-\sqrt{k_{f}}\left(1-\frac{K_{m 11}}{K_{d 11}}\right)}+\frac{\sqrt{k_{f}}\left(\frac{D}{D_{0}}\right)}{1-\sqrt{K_{f}}\left[1-\left(1-\frac{D}{D_{0}}\right) \frac{K_{m 11}}{K_{d 11}}-\left(\frac{D}{D_{0}}\right) \frac{K_{m 11}}{K_{f 11}}\right]}\right\}=K_{\ell 33} \\
& a_{\ell l 1}=k_{m}\left(\frac{E_{m 11}}{E_{\ell l 1}}\right) a_{m 11}+k_{q}\left\{\left[1-\left(\frac{D}{D_{0}}\right)^{2}\right]\left(\frac{E_{d 11}}{E_{\ell l 1}}\right) a_{111}+\left(\frac{D}{D_{0}}\right)^{2}\left(\frac{E_{f l 1}}{E_{\ell l 1}}\right) a_{l 11}\right\} \\
& \alpha_{\ell 22}=\frac{E_{m 22}}{E_{\ell 22}}\left\{\left(1-\sqrt{k_{f}}\right) a_{m 22}+\frac{\left(1-\frac{D}{D_{0}}\right)\left[\left(1-\sqrt{k_{f}}\right) a_{m 22}+\sqrt{k_{f}} a_{d 22}\right]}{1-\sqrt{k_{f}}\left(1-\frac{E_{m 22}}{E_{d 22}}\right)}\right. \\
& \left.+\frac{\sqrt{k_{1}} a_{m 22}-k_{f}\left[a_{m 22}-\left(1-\frac{D}{D_{0}}\right) a_{d 22}-\left(\frac{D}{D_{0}}\right) a_{422}\right]}{1-\sqrt{k_{f}}\left[1-\left(1-\frac{D}{D_{0}}\right) \frac{E_{m 22}}{E_{d 22}}-\left(\frac{D}{D_{0}}\right) \frac{E_{m 22}}{E_{f 22}}\right]}\right\} \cdot a_{233}
\end{aligned}
$$

Figure 4-Micromechanics equations; ply thermal properties. 


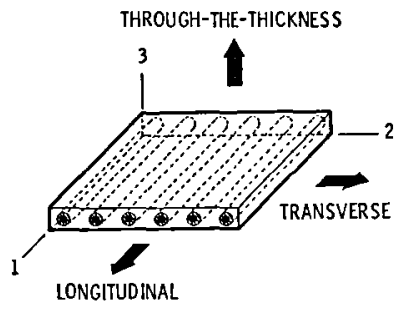

$$
\begin{aligned}
& S_{\ell 11 T}=S_{f l l T}\left\{k_{m}\left(\frac{E_{m l l}}{E_{f l l}}\right)+k_{f}\left[\left(\frac{D}{D_{0}}\right)^{2}+\left\{l-\left(\frac{D}{D_{0}}\right)^{2}\right\} \frac{E_{d l l}}{E_{f l l}}\right]\right\} \\
& \left(S_{f l l c}\left\{k_{m}\left(\frac{E_{m l l}}{E_{f l l}}\right)+k_{f}\left[\left(\frac{D}{D_{0}}\right)^{2}+\left\{1-\left(\frac{D}{D_{0}}\right)^{2}\right\} \frac{E_{d 1 l}}{E_{f 1 l}}\right]\right\}\right. \\
& S_{l 11 C}=\text { MIN. }\left\{S_{m l l C}\left\{k_{m}+k_{f}\left[\left(\frac{D}{D_{0}}\right)^{2} \frac{E_{f 1 l}}{E_{m l l}}+\left\{1-\left(\frac{D}{D_{0}}\right)^{2}\right\} \frac{E_{d 1 l}}{E_{m l l}}\right]\right\}\right. \\
& G_{m 12}\left\{\frac{1}{k_{m}+k_{f}\left[\left(\frac{D}{D_{0}}\right)^{2} \frac{G_{m 12}}{G_{f 12}}+\left\{1-\left(\frac{D}{D_{0}}\right)^{2}\right\} \frac{G_{m 12}}{G_{d 12}}\right]}\right\} \\
& S_{m 11 C}+S_{\ell 12}
\end{aligned}
$$

Figure 5. - Micromechanics equations; ply uniaxial strengths, longitudinal. 


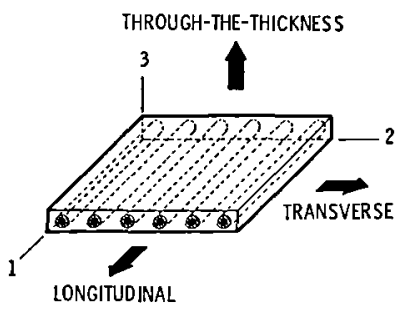

$S_{\text {L22T, } C}=\frac{S_{m 22 T, C}}{\left\{1-\sqrt{k_{f}}\left[1-\left(1-\frac{D}{D_{0}}\right) \frac{E_{m 22}}{E_{d 22}}-\left(\frac{D}{D_{0}}\right) \frac{E_{m 22}}{E_{f 22}}\right]\right\}\left[1+\Phi(\Phi-1)+1 / 3(\Phi-1)^{2}\right]^{1 / 2}}$

WHERE;

$$
0=\frac{1}{\sqrt{\frac{\pi}{4 k_{f}}}-1}\left\{\sqrt{\frac{\pi}{4 k_{f}}}-\frac{\left(\frac{E_{m 22}}{E_{f 22}}\right)}{1-\sqrt{k_{f}}\left[1-\left(1-\frac{D}{D_{0}}\right) \frac{E_{m 22}}{E_{d 22}}-\left(\frac{D}{D_{0}}\right) \frac{E_{m 22}}{E_{f 22}}\right]}\right\}
$$

LOWER BOUND;

$S_{\ell 22 T, C}=\left(1-\sqrt{\frac{\sqrt{k_{f}}}{\pi}}\right) S_{m 22 T, C}$

EQUATIONS FOR INTRALAMINAR SHEAR STRENGTH (S $S_{\ell 12}$ ) ARE ANALOGOUS TO ABOVE EQUATIONS WITH E AND $S_{m 22 T, c}$ REPLACED BY G AND $S_{m 12}$, RESPECTIVELY

Figure 6. - Micromechanics equations; ply uniaxial strengths, transverse and shear.

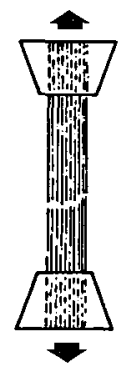

(a) Longitudinal tension.

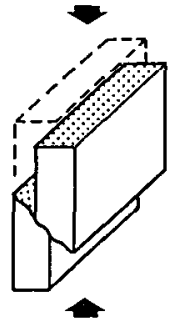

FIBER COMPRESSION MATRIX COMPRESSION

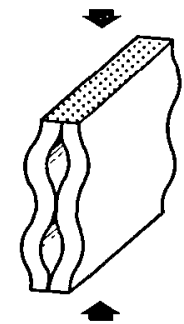

DELAMINATION/ SPLITTING

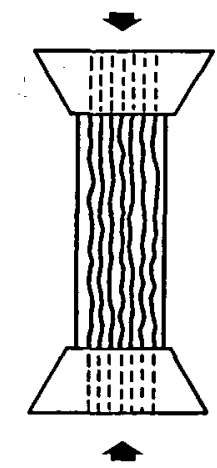

FIBER MICROBUCKLING

(b) Longitudinal compression.

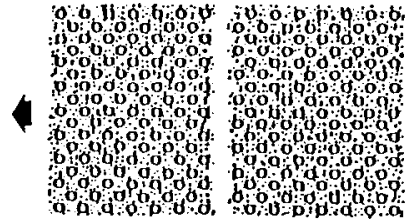

(c) Transverse tension.

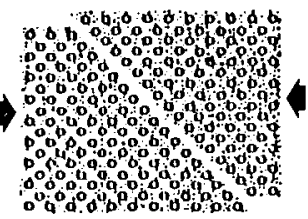

(d) Transverse compression.

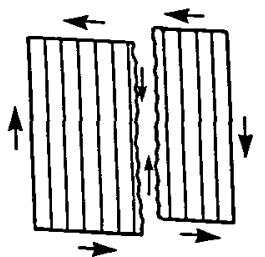

(e) Intralaminar shear.

Figure 7. - In-plane failure modes for unidirectional ply. 


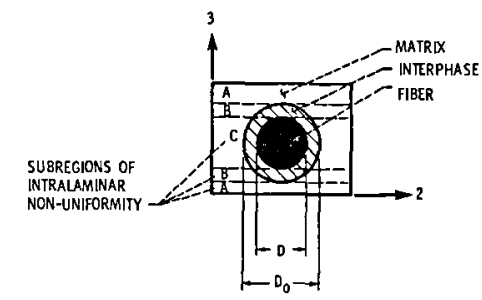

$$
\begin{aligned}
& \sigma_{f 11}=\left[\frac{\sigma_{\ell 11}}{E_{\ell 11}}+\Delta T\left(a_{\ell 11}-a_{f 11}\right)\right] E_{f 11} \\
& \sigma_{f 22}=\frac{E_{f 22}\left\{\frac{\sigma_{\ell 22}}{E_{\ell 22}}+\Delta T\left[a_{\ell 22}-\left(1-\sqrt{k_{f}}\right) \frac{(C)}{(C)}-\sqrt{k_{f}}\left(1-\frac{D}{D_{0}}\right) a_{d 22}-\sqrt{k_{f}}\left(\frac{D}{D_{0}}\right) a_{f 22}\right\}\right.}{1-\sqrt{k_{f}}\left[1-\left(1-\frac{D}{D_{0}}\right) \frac{E_{m 22}}{E_{d 22}}-\left(\frac{D}{D_{0}}\right) \frac{E_{m 22}}{E_{f 22}}\right]} \\
& \sigma_{f 12}=\frac{\left(\frac{\sigma_{\ell 12}}{G_{\ell \ell 2}}\right) G_{f 12}}{1-\sqrt{k_{f}}\left[1-\left(1-\frac{D}{D_{0}}\right) \frac{G_{m 12}}{G_{d 12}}-\left(\frac{D}{D_{0}}\right) \frac{G_{m 12}}{G_{f 12}}\right]}
\end{aligned}
$$

EQUATION FOR $\sigma_{\mathrm{f} 23}$ IS ANALOGOUS TO EQUATION FOR $\sigma_{\mathrm{f} 12}$

Figure 8. - Micromechanics equations; fiber microstresses.

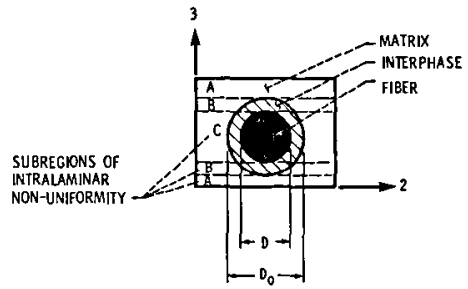

$$
\begin{aligned}
& \sigma_{d 11}=\left[\frac{\sigma_{\ell 11}}{E_{\ell 11}}+\Delta \mathrm{T}\left(a_{\ell 11}-a_{d 11}\right)\right] E_{d 11} \\
& \sigma_{\mathrm{d} 22}^{(B)} \cdot \frac{E_{\mathrm{d} 22}^{(B)}\left\{\frac{\sigma_{\ell 22}}{E_{\ell 22}}+\Delta T\left[a_{\ell 22}-\left(1-\sqrt{k_{\mathrm{f}}}\right) a_{\mathrm{m} 22}^{(B)}-\sqrt{\mathrm{k}_{f}} a_{\mathrm{d} 22}^{(B)}\right]\right\}}{1-\sqrt{\mathrm{k}_{\mathrm{f}}}\left(1-\frac{E_{\mathrm{m} 22}}{E_{\mathrm{d} 22}}\right)}
\end{aligned}
$$

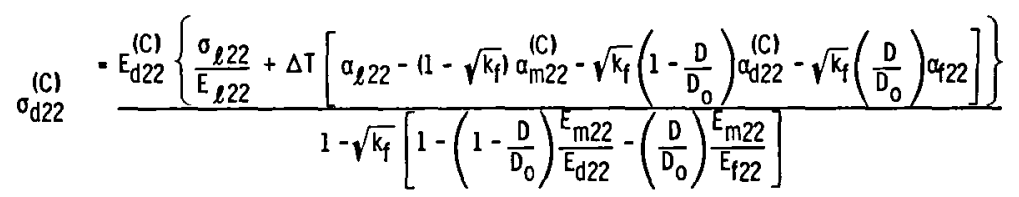

$$
\begin{aligned}
& \sigma_{\mathrm{d} 12}^{(B)} \cdot \frac{\left(\frac{\sigma_{l 12}}{G_{l 12}}\right) G_{\mathrm{d} 12}^{(B)}}{1-\sqrt{k_{f}}\left(1-\frac{G_{\mathrm{ml}}}{G_{\mathrm{d} 12}}\right)}
\end{aligned}
$$

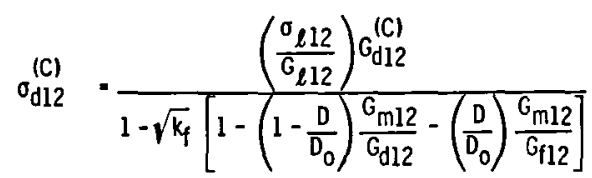

$$
\begin{aligned}
& \text { EQUATIONS FOR } \sigma_{\mathrm{d} 23}^{(\mathrm{B}, \mathrm{C})} \text { ARE ANALOGOUS TO EQUATIONS FOR } \sigma_{\mathrm{d} 12}^{(\mathrm{B}, \mathrm{Cl}}
\end{aligned}
$$

Figure 9. - Micromechanics equations; interphase microstresses. 


$$
\begin{aligned}
& \text { SUBREGINS OR } \\
& \sigma_{m l l} \quad \cdot\left[\frac{\sigma_{\ell 11}}{E_{\ell 11}}+\Delta T\left(a_{\ell 11}-a_{m 11}\right)\right] E_{m l l} \\
& \sigma_{m 22}^{(A)}-\left[\frac{\sigma_{\ell 22}}{E_{\ell 22}}+\Delta \mathrm{T}\left(a_{\ell 22}-a_{m 22}^{(A)}\right)\right] E_{m 22}^{(A)} \\
& \sigma_{m 22}^{(B)} \cdot \frac{E_{m 22}^{(B)}\left\{\frac{\sigma_{\ell 22}}{E_{\ell 22}}+\Delta T\left[a_{\ell 22}-\left(1-\sqrt{\left.k_{f}\right)} a_{m 22}^{(B)}-\sqrt{k_{f}} a_{d 22}^{(B)}\right]\right\}\right.}{1-\sqrt{k_{f}}\left(1-\frac{E_{m 22}}{E_{d 22}}\right)}
\end{aligned}
$$

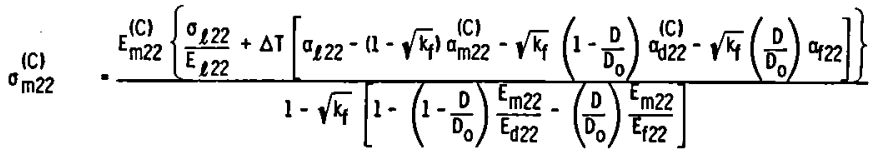

$$
\begin{aligned}
& \underset{\sigma_{\mathrm{m} 12}^{(A)}}{A^{2}} \cdot\left(\frac{\sigma_{\ell 12}}{\sigma_{l 12}}\right) G_{\mathrm{ml2}}^{(A)} \\
& \sigma_{m 12}^{(B)} \quad \cdot \frac{\left(\frac{\sigma_{\ell 12}}{G_{\ell 12}}\right) G_{m 12}^{(B)}}{1-\sqrt{k_{f}}\left(1-\frac{G_{m 12}}{G_{d 12}}\right)} \\
& \sigma_{m 12}^{(C)} \cdot \frac{\left(\frac{\sigma_{\ell 12}}{\sigma_{\ell 12}}\right) G_{m 12}^{(C)}}{1-\sqrt{\mathrm{t}_{1}}\left[1-\left(1-\frac{D}{D_{0}}\right) \frac{G_{m 12}}{G_{d 12}}-\left(\frac{D}{D_{0}}\right) \frac{G_{m 12}}{G_{112}}\right]}
\end{aligned}
$$

Figure 10. - Micromechanics equations; matrix microstresses. 


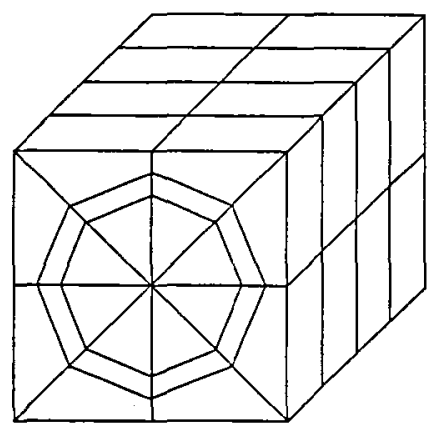

FINITE ELEMENT MODEL

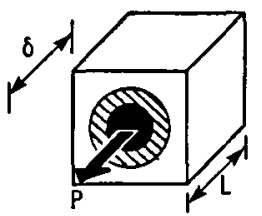

$E=\frac{P L}{A \delta}$

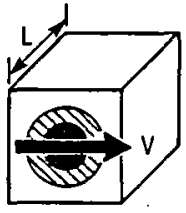

$G=\frac{V L}{A r}$

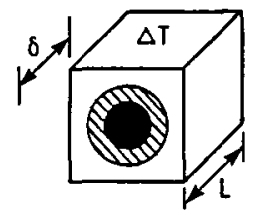

$a=\frac{\delta}{(\Delta T) L}$

Figure 11. - Micromechanics/finite element validation; finite element model and simple mechanics of materials expressions for idealized deformation modes.
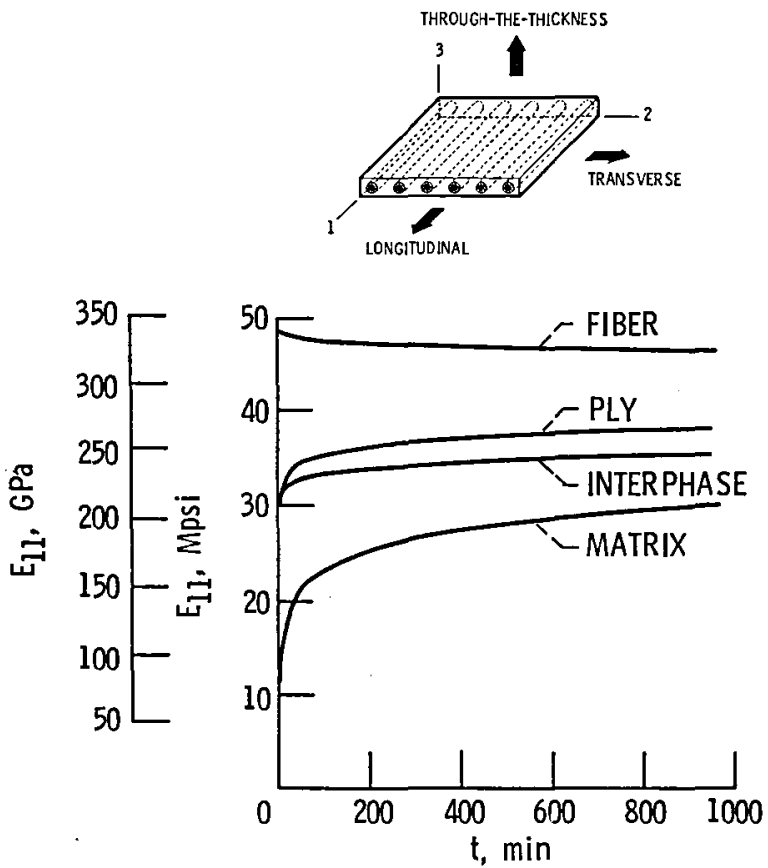

Figure 12. - Fabrication cool-down transient; variation of $E_{11}$ for constituents and ply. 


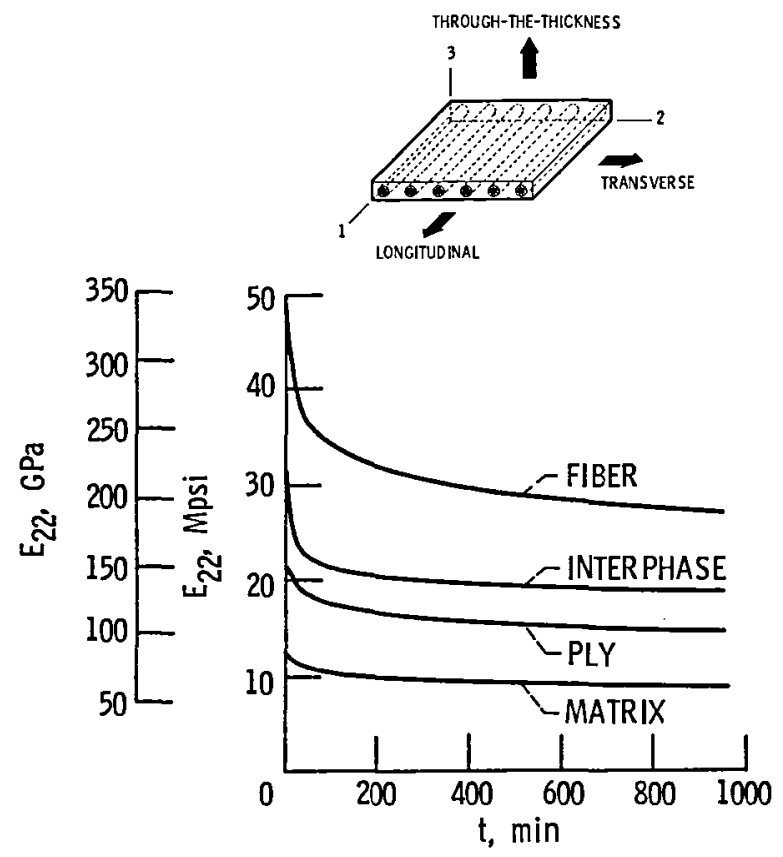

Figure 13. - Fabrication cool-down transient; variation of $E_{22}$ for constituents and ply.
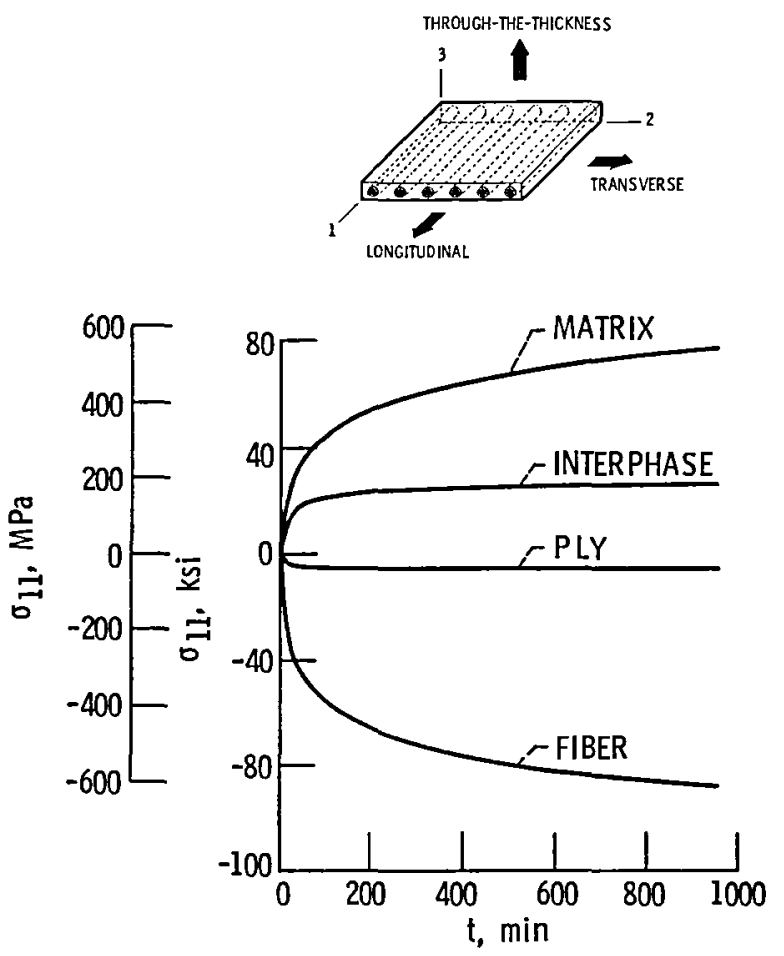

Figure 14. - Fabrication cool-down transient; induced residual stress $\left(\sigma_{11}\right)$ for ply and constituents. 


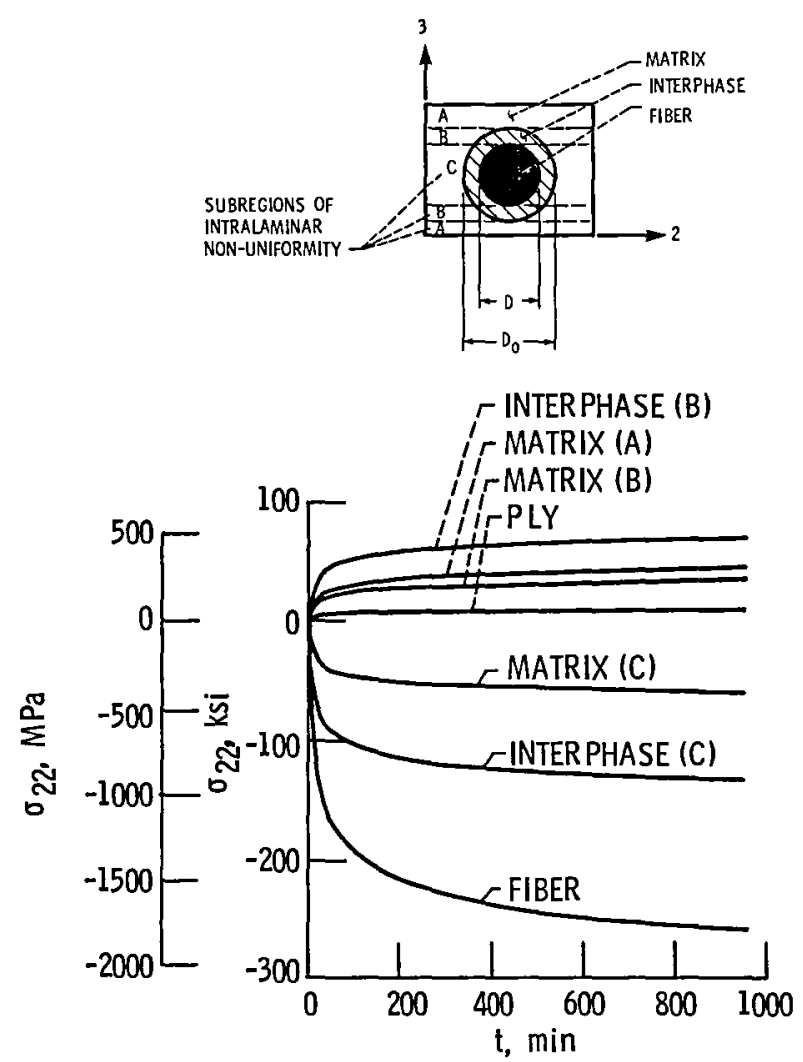

Figure 15. - Fabrication cool-down transient; induced residual stress $\left(\sigma_{22}\right)$ for ply and constituents. 


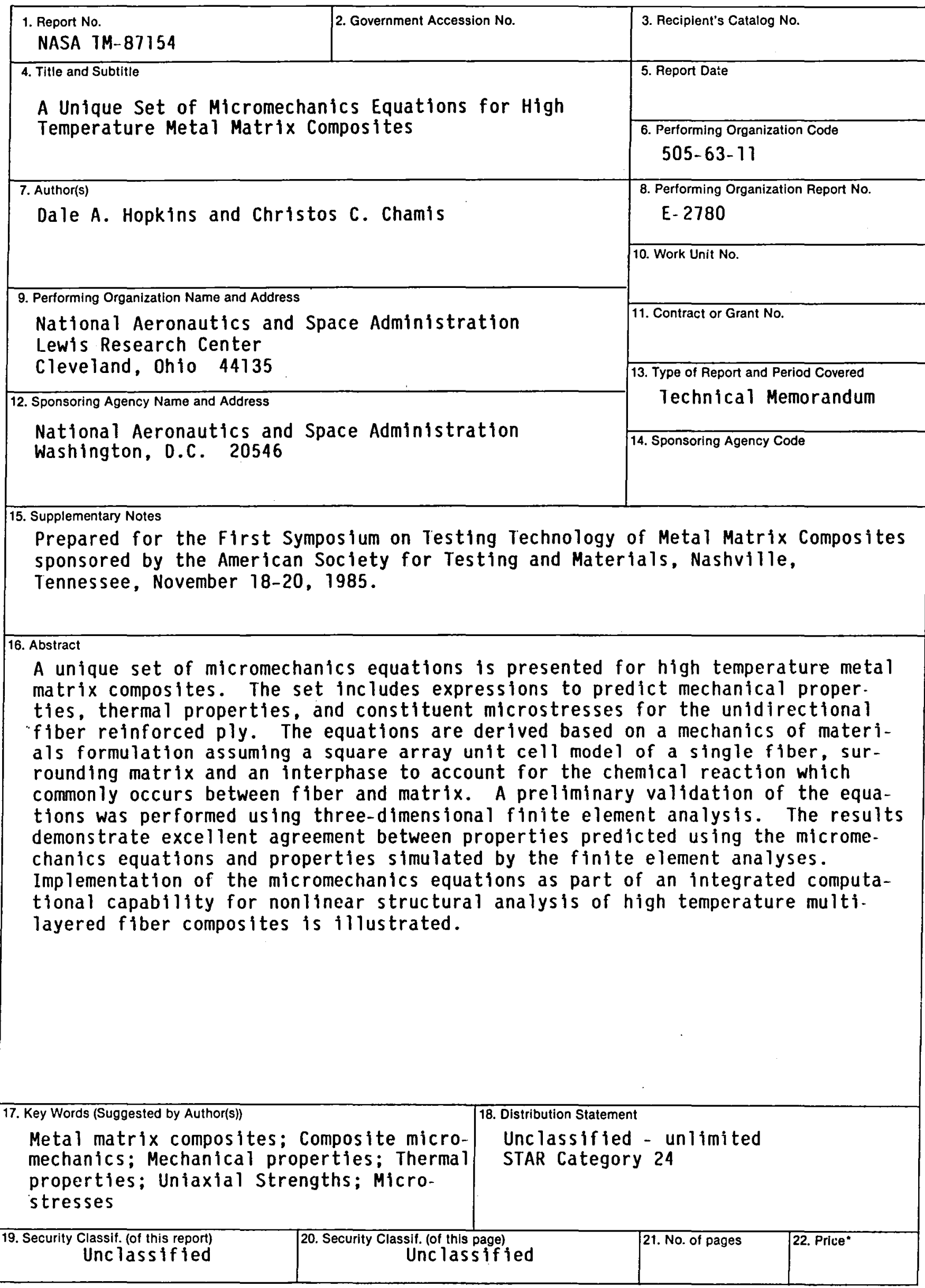


ERRATA

NASA Technical Memorandum 87154

\section{A UNIQUE SET OF MICROMECHANICS EQUATIONS FOR HIGH TEMPERATURE METAL MATRIX COMPOSITES}

Dale A. Hopkins and Christos C. Chamis November 1985

The following corrections apply to the appendix and occur on page 13:

1. The denominator of Equation (12) should read as follows:

$$
\left\{1-\sqrt{k_{f}}\left[1-\left(1-\frac{D}{D_{0}}\right)\left(\frac{E_{m}}{E_{d}}\right)-\left(\frac{D}{D_{0}}\right)\left(\frac{E_{m}}{E_{f}}\right)\right]\right\}
$$

2. The first full sentence after Equation (12) should read as follows:

"The equivalent modulus for subregion $B$ is deduced from Eq. (12) by letting $0 / D_{0}$ equal zero."

3. The denominator of Equation (13) should read as follows:

$$
\left\{1-\sqrt{k_{f}}\left[1-\left(\frac{E_{m}}{E_{d}}\right)\right]\right\}
$$

4. The sentence after Equation (15) should read as follows:

"Dividing through by $S_{\ell}$, substituting the definitions from

Eqs. (7) and (8) and the results from Eqs. (12) through (14), and rearranging gives"

5. The denominator of the second term inside the braces on the right-hand side of Equation (16) should read as follows:

$$
1-\sqrt{k_{f}}\left[1-\left(\frac{E_{m}}{E_{d}}\right)\right]
$$


ERRATA

NASA Technical Memorandum 87154

A UNIQUE SET OF MICROMECHANICS EQUATIONS FOR HIGH TEMPERATURE METAL MATRIX COMPOSITES

Dale A. Hopkins and Christos C. Chamis November 1985

The following corrections apply to the appendix and occur on page 13:

1. The denominator of Equation (12) should read as follows:

$$
\left\{1-\sqrt{k_{f}}\left[1-\left(1-\frac{D}{D_{0}}\right)\left(\frac{E_{m}}{E_{d}}\right)-\left(\frac{D}{D_{0}}\right)\left(\frac{E_{m}}{E_{f}}\right)\right]\right\}
$$

2. The first full sentence after Equation (12) should read as follows:

"The equivalent modulus for subregion $B$ is deduced from Eq. (12) by letting $\mathrm{D} / \mathrm{D}_{0}$ equal zero."

3. The denominator of Equation (13) should read as follows:

$$
\left\{1-\sqrt{k_{f}}\left[1-\left(\frac{E_{m}}{E_{d}}\right)\right]\right\}
$$

4. The sentence after Equation (15) should read as follows:

"Dividing through by $S_{\ell}$, substituting the definitions from Eqs. (7) and (8) and the results from Eqs. (12) through (14), and rearranging gives"

5. The denominator of the second term inside the braces on the right-hand side of Equation (16) should read as follows:

$$
1-\sqrt{k_{f}}\left[1-\left(\frac{E_{m}}{E_{d}}\right)\right]
$$




\section{End of Document}

